\title{
Chapter 10 \\ Simulation of Gross Domestic Product \\ in International Trade Networks: Linear \\ Gravity Transportation Model
}

\author{
Tsuyoshi Deguchi, Hideki Takayasu, and Misako Takayasu
}

\begin{abstract}
In this study, we introduce a model to simulate gross domestic product (GDP) for international trade network data. By applying a linear gravity transportation model, we confirm that estimated values approximately agree with the real values of GDP by tuning the model parameters. An exception is the estimated GDP of China that is about two times bigger than the real value. This discrepancy might imply that China's GDP is not saturated and it is on the way of growing.
\end{abstract}

\subsection{Introduction}

Today, China is becoming increasingly influential in not only the international community but also international politics and military forces. However, its presence is most notable in the international economy. In particular, with China's growing gross domestic product (GDP), the country has the potential to become the biggest economy in the near future. GDP is the most specific and popular measure in the economic statistics literature, although many have doubted China's GDP statistics $[1,2]$.

\footnotetext{
T. Deguchi $(\bowtie) \bullet$ M. Takayasu

Department of Computational Intelligence and Systems Science, Interdisciplinary Graduate

School of Science and Engineering, Tokyo Institute of Technology, 4259 Nagatsuta-cho,

Midori-ku, Yokohama 226-8502, Japan

e-mail: deguchi.t.ad@m.titech.ac.jp; takayasu@dis.titech.ac.jp

H. Takayasu

Sony Computer Science Laboratories, 3-14-13 Higashigotanda, Shinagawa-ku, Tokyo 141-0022, Japan

Graduate School for Advanced Mathematical Sciences, Meiji University, 4-21-1 Nakano, Nakano-ku, Tokyo 164-8525, Japan

Department of Computational Intelligence and Systems Science, Interdisciplinary Graduate School of Science and Engineering, Tokyo Institute of Technology, 4259 Nagatsuta-cho, Midori-ku, Yokohama 226-8502, Japan

e-mail: takayasu@csl.sony.co.jp
}

(C) The Author(s) 2015

H. Takayasu et al. (eds.), Proceedings of the International Conference on Social

Modeling and Simulation, plus Econophysics Colloquium 2014, Springer

Proceedings in Complexity, DOI 10.1007/978-3-319-20591-5_10 
In this paper, we estimate and simulate countries' GDP and ranks using the linear gravity transportation model (LGTM). The gravity transportation model (GTM) is known to be effective in examining a company's transaction networks $[3,4]$. The LGTM is a linearized form of the GTM and is a type of degree distribution model $[5,6]$.

\subsection{Preceding Study}

International trade networks (ITNs) are predominantly used in surveying network structures and known to follow the so-called "gravity relation" [7]. They were first examined in 2003 by Serrano and Boguñá, who presented the fundamental characteristics of ITNs for different countries [8]. Recently, physicists and network researchers have explored the structure on the basis of diverse factors, such as time series robustness, community structures, and inter-layer dependency [9-12]. Some researchers have attempted to extend ITN research to that on economic growth [13-16]. These studies contribute some interesting findings from the viewpoint of complex networks. For instance, Garlaschelli et al. found that GDP is a hidden factor that influences networks [17]. Although this fact is common knowledge in international economics, their model remains an impressive contribution to network study. Several other studies have been conducted on gravity relations [18-20].

\subsection{Dataset}

We adopt data from the Direction of Trades Statistics (DOTS) compiled by the International Monetary Fund (IMF) [21]. This dataset includes annual and monthly data of trades (US dollar) for countries. We use a total of 214 countries (regions) as nodes and their respective trade amounts as weighted links. The weighted links suggest bilateral trade relationships, that is, exports and imports, between countries or regions on a monthly and yearly basis, which are measured in million US dollar. DOTS also include the base data for both exports and imports. In general, the amount of export from country A to country B should be same as the amount of import to country B from country A. However, these numbers differ between the import base and export base datasets. In this case, we use the export base year dataset.

We also use GDP data from the Economic Outlook Dataset, also produced by IMF. We use this data with enough credit. However, this dataset includes only 189 countries. Thus, we arrange these datasets and aggregate ITN and GDP data.

After data processing, we used 2010 ITN and GDP data for 160 countries. 


\subsection{Simulation Setup}

\subsubsection{GDP Transaction Flow Relationship}

First, we define ITN as an adjacency matrix, $W$, whose component, $w_{i j}$, represents the annual amount of transaction flow (imports) from country $i$ to country $j$, measured in million US dollars. Then, we define $w_{i i}=0$. We also introduce the binary network matrix, $A$, whose component $a_{i j}=1$ when $w_{i j}>0$ and $a_{i j}=0$ when $w_{i j}=0$.

The following relationship between GDP and transaction flows is often assumed in the international trade literature[7].

$$
w_{i j}=G \frac{Y_{i}^{\alpha} Y_{j}^{\beta}}{R_{i j}^{\gamma}} .
$$

Here, $Y_{i}$ is the GDP of node $i$ and $R_{i j}$ is the distance between nodes $i$ and $j$. The power exponent $\alpha, \beta$, and $\gamma$ are parameters. We neglect the distance $R_{i j}$ and estimate the exponents $\alpha$ and $\beta$ using data shown in Fig. 10.1.

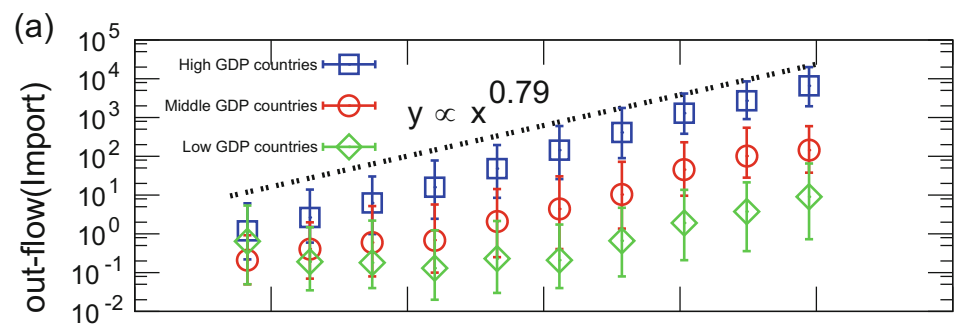

(b)

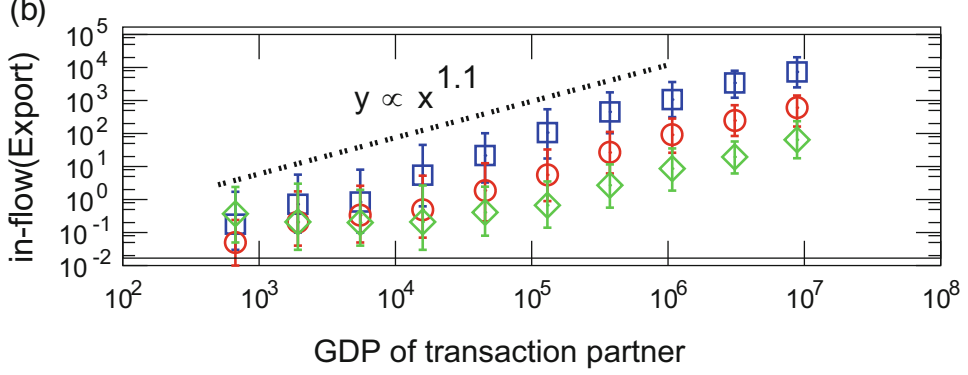

Fig. 10.1 GDP Transaction flow (import and export) relationship in a log-log plot. (a) Import (b) Export for high GDP countries (top one-third of high GDP countries), middle GDP countries (other countries not classified as high and low GDP countries), and low GDP countries (bottom one-third of high GDP countries). In this case, high, middle, and low GDP relates to the total GDP. Here, bins are defined at regular intervals in log-scale, the first and third quantiles are plotted as error bars with the median value at the center of symbols, squares and circles 
Fig. 10.2 GDP-degree relationship in a log-log plot. The first and third quantiles are plotted as error bars with the median value at the center of squares

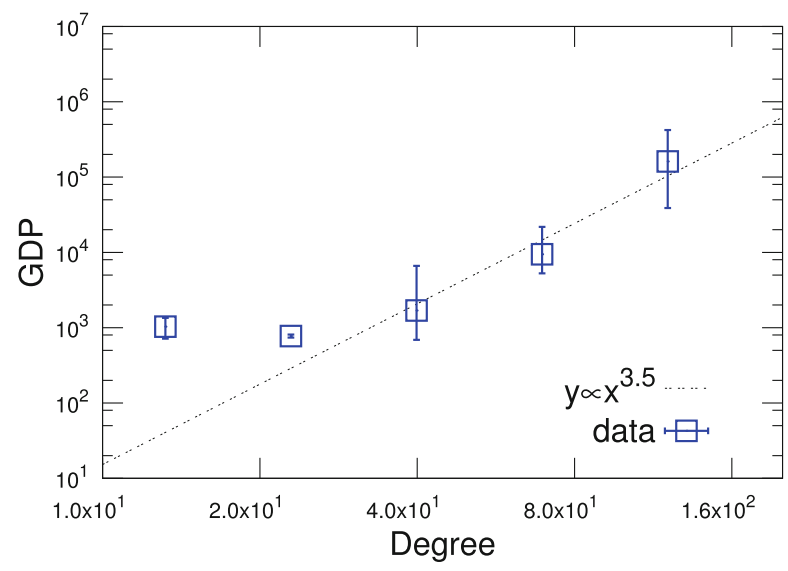

$$
w_{i j} \propto Y_{i}^{\alpha} Y_{j}^{\beta}
$$

Using parameter fitting, we estimate the values of $\alpha$ and $\beta$ as $(\alpha, \beta)=$ $(0.79,1.1)$.

\subsubsection{GDP-Degree Relationship}

The number of trade parameters is higher for high GDP countries. We confirm the following power law relationship between the degrees and GDPs (Fig. 10.2). Here, we define the in-degree as $k_{M}=\sum_{i} a_{i M}$.

$$
Y_{M} \propto k_{M}^{\zeta}
$$

Using the data, we estimate $\zeta=3.5$ (Fig. 10.2).

\subsubsection{Linear Gravity Transportation Model}

From Figs. 10.1 and 10.2, we know that the amount of trades, degrees and GDPs have positive relations. Thus, we model these relations as a transportation model. In this model, we think the number of degrees directly affects the amount of trade flow, where in-degree is defined as $k_{j}=\sum_{i} a_{i j}$. And we calculate the GDPs as a result of the distributions of trades flows using gravity relation. We call this model as a LGTM, which is conceptually depicted in Fig. 10.3. 
Fig. 10.3 Conceptual figure of weights of out-flow from a node in LGTM. In this case, the amounts proportional to GDPs are transported and distributed to neighbor nodes that are proportional to the $\omega$-th power of other nodes' in-degrees

Fig. 10.4 Conceptual figure of all in-flows and out-flows for a node $\mathrm{M}$ in LGTM. The black arrows denote inflow-outflow relationships among nodes and the dotted ones show flow relationships outside of the network
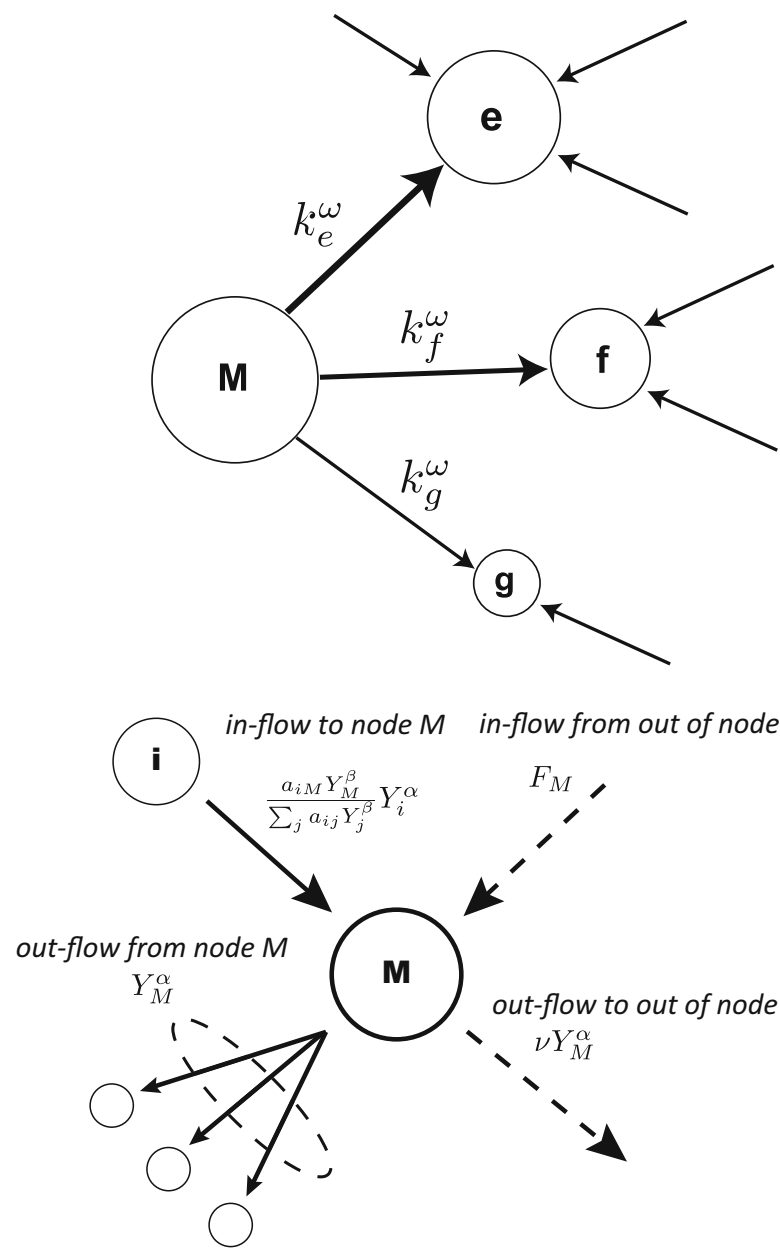

LGTM is based on four types of flows. In the case of node $M$, inflow in an inter-node relationship is defined as $\frac{a_{i M} k_{M}^{\omega}}{\sum_{j} a_{i j} k_{j}^{\omega}} Y_{i}^{\alpha}$, which is affected by the degrees. Total outflow from node $\mathrm{M}$ in the inter-node relationship is $Y_{M}^{\alpha}$. Inflow and outflow outside of the inter-node relationship are $F_{M}$ and $v Y_{M}^{\alpha}$, respectively (Fig. 10.4). In the equilibrium, the aggregation of all flows is assumed to be zero.

$$
\sum_{i} \frac{a_{i M} k_{M}^{\omega}}{\sum_{j} a_{i j} k_{j}^{\omega}} Y_{i}^{\alpha}-(1+v) Y_{M}^{\alpha}+F_{M}=0 .
$$




\subsection{Simulation Results}

Using LGTM, we estimate the GDPs for the given ITN data. First, we must acquire LGTM's parameters $\left(\alpha, \omega, v, F_{M}\right)$ from the real data's parameter fitting. Then, we estimate $\alpha$ and $\omega$ by minimizing the following function:

$$
F(\alpha, \omega)=\sum_{i} \sum_{M}\left\{\log \left(\frac{w_{i M}}{\frac{a_{i M} k_{M}^{\omega}}{\sum_{j} a_{i j} k_{j}^{\omega}} Y_{i}^{\alpha}}\right)\right\}^{2} .
$$

Next, we derive $v$ from the inflow-outflow relationship in Eq. (10.6) as a transformation of equilibrium (10.4). Here, $Y_{M}^{\alpha}$ equals outflow (imports) and $\sum_{i} \sum_{i{ }_{j} k_{i j} k_{j}^{\omega}} Y_{i}^{\alpha}$ equals inflow (exports). Thus, we get $\frac{1}{(1+v)}$ as the regression coefficient.

$$
Y_{M}^{\alpha}=\frac{1}{(1+v)} \sum_{i} \frac{a_{i M} k_{M}^{\omega}}{\sum_{j} a_{i j} k_{j}^{\omega}} Y_{i}^{\alpha}+\frac{F_{M}}{(1+v)} .
$$

Therefore, we get $F_{M}$ from the equilibrium in Eq. (10.7).

$$
F_{M}=\frac{v \sum_{i} Y_{M}^{\alpha}}{N}
$$

We obtain the values as $\left(\alpha, \omega, v, F_{M}\right)=\left(0.68,4.5,0.032,1.3 \times 10^{2}\right)$.

In the case of ITN, there are little differences among top countries' degrees. We introduce the preferentially selected network [6]—a simplified network produced from the original one, in which links with small contributions are removed using the following rule: For all nodes, we select the top $n^{\text {th }}$ weight links for both inflow and outflow and cut off all other links. In this simulation, we use the case of preferentially selected network, where $n=6$.

Under these conditions, we estimate GDP using Eq. (10.6) (Fig. 10.5). We find that the results between the real and simulated GDPs are fairly close. If the real and simulated values are proportionate, the values are assumed to be on the 45 degree line.

Next, we check the top 20 countries for both the simulated and real GDP. The results are listed in Table 10.1. In the real data, China ranks second, whereas in the simulation, it is at the top. China's estimated GDP is about two times bigger than the real GDP. This discrepancy might be caused by slow time evolution that China's GDP is on the way of approaching to the equilibrium value which is determined by the trading network structure. Simulation results are based on the equilibrium and China's GDP would be bigger in near future from the viewpoint of trade. 
Fig. 10.5 Real and simulated GDPs estimated using Eq. (10.6) and preferentially selected network $(n=6)$. The first and third quantiles are plotted as error bars with the median value at the center of squares

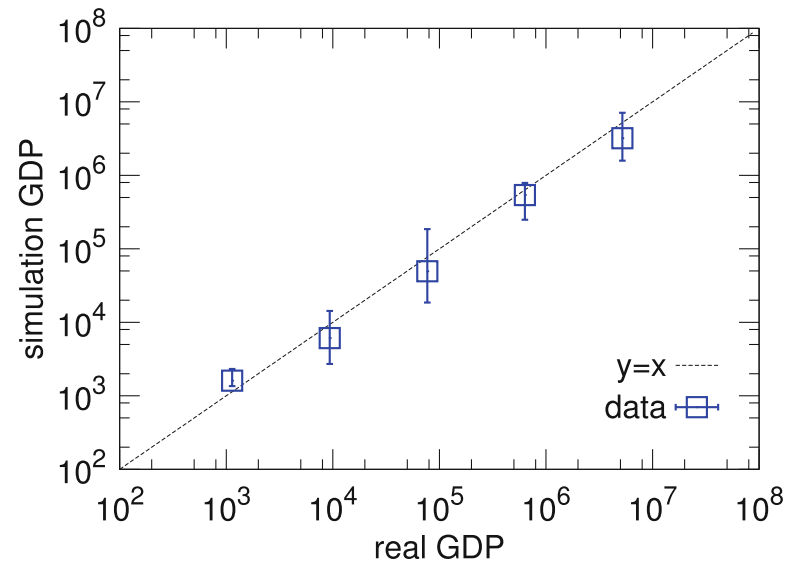

Table 10.1 Top 20 countries (real and simulated data)

\begin{tabular}{l|l|l|l|l}
\hline Country & Real GDP & Simulated GDP & Real rank & Simulated rank \\
\hline United States & $1.5 \times 10^{7}$ & $8.2 \times 10^{6}$ & 1 & 2 \\
\hline China & $5.9 \times 10^{6}$ & $1.2 \times 10^{7}$ & 2 & 1 \\
\hline Japan & $5.5 \times 10^{6}$ & $4.7 \times 10^{6}$ & 3 & 4 \\
\hline Germany & $3.3 \times 10^{6}$ & $6.7 \times 10^{6}$ & 4 & 3 \\
\hline France & $2.7 \times 10^{6}$ & $1.7 \times 10^{6}$ & 5 & 6 \\
\hline United Kingdom & $2.3 \times 10^{6}$ & $1.5 \times 10^{6}$ & 6 & 9 \\
\hline Brazil & $2.1 \times 10^{6}$ & $1.4 \times 10^{6}$ & 7 & 11 \\
\hline Italy & $2.1 \times 10^{6}$ & $1.6 \times 10^{6}$ & 8 & 8 \\
\hline India & $1.7 \times 10^{6}$ & $1.2 \times 10^{6}$ & 9 & 12 \\
\hline Canada & $1.6 \times 10^{6}$ & $5.9 \times 10^{5}$ & 10 & 22 \\
\hline Russia & $1.5 \times 10^{6}$ & $8.8 \times 10^{5}$ & 11 & 16 \\
\hline Spain & $1.4 \times 10^{6}$ & $3.9 \times 10^{5}$ & 12 & 33 \\
\hline Australia & $1.2 \times 10^{6}$ & $1.1 \times 10^{6}$ & 13 & 15 \\
\hline Korea & $1.1 \times 10^{6}$ & $2.4 \times 10^{6}$ & 14 & 5 \\
\hline Mexico & $1.1 \times 10^{6}$ & $1.0 \times 10^{4}$ & 15 & 95 \\
\hline Netherlands & $8.4 \times 10^{5}$ & $1.7 \times 10^{6}$ & 16 & 7 \\
\hline Turkey & $7.3 \times 10^{5}$ & $5.7 \times 10^{5}$ & 17 & 24 \\
\hline Indonesia & $7.1 \times 10^{5}$ & $1.1 \times 10^{6}$ & 18 & 13 \\
\hline Switzerland & $5.5 \times 10^{5}$ & $6.0 \times 10^{5}$ & 19 & 21 \\
\hline Saudi Arabia & $5.3 \times 10^{5}$ & $4.5 \times 10^{5}$ & 20 & 30 \\
\hline
\end{tabular}

In this simulation, we use parameters $\left(\alpha, \omega, v, F_{M}\right)$ in three significant digits and simulation results are represented in two significant digits

\subsection{Conclusion}

In this paper, we empirically introduced a linear gravity transportation model of world trade based on the network structure among countries. By tuning the model's parameters, we confirmed that estimated values approximately agree with the real 
values of GDP. One apparent exception is China that its estimated GDP value is about two times bigger than the real value. This discrepancy might imply that China's GDP is growing rapidly and the steady state solution of our model for given world trade network structure does not fit well.

Acknowledgements This work was supported by a Grant-in-Aid for Scientific Research (B), Grant Number 26310207 and a Grant-in-Aid for Scientific Research (C), Grant Number 24540395.

Open Access This book is distributed under the terms of the Creative Commons Attribution Noncommercial License which permits any noncommercial use, distribution, and reproduction in any medium, provided the original author(s) and source are credited.

\section{References}

1. Rabinovitch S, Chinese econ stats: to doubt or not to doubt. (Financial Times, 28 June 2012), http://blogs.ft.com/beyond-brics/2012/06/28/china-stats-to-doubt-or-not-to-doubt. Accessed 30 Jan 2015

2. Rawski T (2001) China Econ Rev 12:347-354

3. Tamura K, Miura W, Takayasu H, Kitajima S, Goto H, Takayasu M (2012) Proceedings of the Asia Pacific symposium on intelligent and evolutionary systems, Kyoto, Japan. (ISBN978-499066920-1, 2012)

4. Tamura K, Miura W, Takayasu M, Takayasu H, Kitajima S, Goto H (2012) Int J Mod Phys Conf Ser 16:93-104

5. Watanabe H, Takayasu H, Takayasu M (2012) New J Phys 14:043034

6. Deguchi T, Takayasu H, Takayasu M. In preparation

7. Krugman P, Obstfeld M, Melitz M (2011) International economics: theory and policy, 9th edn. Pearson Education, Boston

8. Serrano M, Boguñá M (2003) Phys Rev E 68:015101

9. Garlaschelli D, Loffredo M (2005) Physica A 355(1):138-144

10. Fagiolo G, Reyes J, Schiavo S (2009) Phys Rev E 79:036115

11. Tzekina I, Danthi K, Rockmore D (2010) Eur Phys J B 63:541-545

12. Barigozzi M, Fagiolo G, Garlaschelli D (2010) Phys Rev E 81:046104

13. Hidalgo C, Klinger B, Barabási A, Hausmann R (2007) Science 317:482-487

14. Hidalgo C, Hausmann R (2009) Proc Natl Acad Sci 106:10570-10575

15. Tacchella A, Cristelli M, Caldarelli G, Gabrielli A, Pietronero L (2012) Sci Rep 2:723

16. Zaccaria A, Cristelli M, Tacchella A, Pietronero L PloS one (2014) 9:e113770

17. Garlaschelli D, Loffredo M (2004) Phys Rev Lett 93:188701

18. Fagiolo G (2010) J Econ Interact Coord 5:1-25

19. Dueñas M, Fagiolo G (2013) J Econ Interact Coord 8:155-178

20. Almog A, Squartini T, Garlaschelli D (2015) New J Phys 17:013009

21. Direction of trade statistics. In: IMF eLibrary data. http://elibrary-data.imf.org. Accessed 30 Jan 2015 